\title{
Exact method to solve of linear heat transfer problems
}

\author{
Akmaljon Abdullayev", Kholsaid Kholturayev and Nigora Safarbayeva \\ Tashkent Institute of Irrigation and Agricultural Mechanization Engineers, Tashkent, Uzbekistan
}

\begin{abstract}
When approximating multidimensional partial differential equations, the values of the grid functions from neighboring layers are taken from the previous time layer or approximation. As a result, along with the approximation discrepancy, an additional discrepancy of the numerical solution is formed. To reduce this discrepancy when solving a stationary elliptic equation, parabolization is carried out, and the resulting equation is solved by the method of successive approximations.

This discrepancy is eliminated in the approximate analytical method proposed below for solving two-dimensional equations of parabolic and elliptic types, and an exact solution of the system of finite difference equations for a fixed time is obtained.

To solve problems with a boundary condition of the first kind on the first coordinate and arbitrary combinations of the first, second and third kinds of boundary conditions on the second coordinate, it is proposed to use the method of straight lines on the first coordinate and ordinary sweep method on the second coordinate. Approximating the equations on the first coordinate, a matrix equation is built relative to the grid functions. Using eigenvalues and vectors of the three-diagonal transition matrix, linear combinations of grid functions are compiled, where the coefficients are the elements of the eigenvectors of the three-diagonal transition matrix.

Boundary conditions, and for a parabolic equation, initial conditions are formed for a given combination of grid functions. The resulting onedimensional differential-difference equations are solved by the ordinary sweep method. From the resulting solution, proceed to the initial grid functions.

The method provides a second order of approximation accuracy on coordinates. And the approximation accuracy in time when solving the parabolic equation can be increased to the second order using the central difference in time.

The method is used to solve heat transfer problems when the boundary conditions are expressed by smooth and discontinuous functions of a stationary and non-stationary nature, and the right-hand side of the equation represents a moving source or outflow of heat.
\end{abstract}

*Corresponding author: akma109.07.85@mail.ru 


\section{Introduction}

The development of the theory of heat transfer can be traced by publications and republications of books "Heat Transfer" by Isachenko V.P., Osipova V.A., Sukomel A.S., "Theory of heat and mass exchange" [1], "Theory of heat and mass transfer" by Lykov A.V., Mikhailov Yu.A. [2], "Computational heat transfer" by Samarsky A.A., Vabishchevich P.N. [3], and other publications in the subject area.

In [4], the classification of methods (variation, linearization, projection, etc.) for solving non-stationary nonlinear heat and mass transfer problems in solids was carried out. For linear problems, traditional methods of variables separation, sources, Laplace, LaplaceCarson, Fourier, Hankel and other methods are used. The solutions to numerous heat transfer problems with different boundary conditions, including the mixed ones, are given in single and multilayer materials using the generalized variables. Some problems have been solved, taking into account the freezing of soil and building materials.

Yang Chen, Qie Sun and Ronald Wennersten in [5] discussed the results of an experimental study of boiling water flow inside a vertical mini-channel. The thermal conductivity coefficients were obtained for various values of mass flow $\left(5-58 \mathrm{~kg} / \mathrm{m}^{2} \mathrm{~s}\right)$ and heat flow $\left(1-5.6 \mathrm{~kW} / \mathrm{m}^{2}\right)$. The effect of heat flow, mass flow and steam quality on the coefficient of thermal conductivity was analyzed. Based on the existing three-zone model, a new theoretical model has been developed for calculating the thermal conductivity coefficient of the bubble part in the mini-channel. With an absolute error of $9.72 \%$, the new model provides a good agreement with experimental data.

Wenke Zhang et al. [6] analyzed the heat transfer process between the ground heat exchangers of surface systems and a heat pump. The system operation is based on the concept that the ground medium is a source of heat or cold, and the heat pump supplies heat or cold from the ground heat exchangers to the air-conditioned room. A well-grounded analysis of the system makes it possible to optimally construct and locate the ground heat exchangers and minimize their costs. Here, it is necessary to consider such factors as location, the volume of heat exchangers, type of circulating fluid and others. The heat exchange processes inside and outside the boreholes with heat exchangers were analyzed.

The paper by Chungen Yin [7] is devoted to the study of convective heat transfer processes and the evaporation of moving fuel droplets. An integrated three-dimensional model has been developed, consisting of the circulation of the internal flow, heat and mass transfer in a moving fuel droplet. The model is calibrated by analytical solutions for simplified cases.

Among works published in the 21 st century, the monograph by P.V. Tsoi [8] has a significant value for the theory of heat and mass transfer. The methods of Laplace integral transforms, Carson-Laplace, Fourier and others are widely studied in this research work. In combination with the Galerkin projective method, the author managed to create high accuracy methods to solve the direct and inverse heat and mass transfer problems in Cartesian, cylindrical and spherical coordinates. The solved problems are expanded for cylindrical bodies and channels of complex profile (with a single and double connected cross-section).

In numerous literary sources, one can find Stefan's problems in various statements and solutions [8-11], solved by numerical methods. Recent works on numerical methods are presented in $[2,12-16]$.

As the analysis of numerical methods for solving heat transfer problems, particularly partial differential equations, shows that the main drawback is the inconsistency of the grid functions from neighboring layers used in approximation. In particular, in [13-14], to solve the equation for hydrostatic pressure of an elliptical type, a fictitious time is introduced, and a solution is established. 
This is because in sweeping on one of the coordinates, grid functions from neighboring layers are used, determined from the previous time layer; they form the discrepancies in the solution. Below, on the example of solving two-dimensional problems of elliptic and parabolic types of equations, a numerical method is demonstrated that allows us to eliminate these discrepancies and obtain an exact solution of finite difference equations when the boundary conditions of the first kind are on the first coordinate and an arbitrary combination of the boundary conditions of the first, second and third kinds are on the other coordinate.

The approximate analytical method for solving equations, proposed below, is a symbiosis of the method of straight lines on the coordinate, the conditions of which belong to the first kind [15-16], and the ordinary sweep method - for a combination of arbitrary boundary conditions [3,13-14]. The capabilities and features of the method are demonstrated by the example of stationary and non-stationary heat transfer problems.

Statement of the problem. Two-dimensional heat transfer process is described by equation [3].

$$
\frac{\partial T}{\partial t}=a^{2}\left(\frac{\partial^{2} T}{\partial x^{2}}+\frac{\partial^{2} T}{\partial y^{2}}\right)+f(x, y, t)
$$

where $t$ is time; $x, y$ are Cartesian coordinates; $a^{2}$ is coefficient of thermal conductivity of the material; $f(x, y, t)$ are the total intensity of internal and external heat sources at point $(x, y)$, given by the product of heat capacity and density of the material.

The initial temperature distribution in the calculation domain is set

$$
T(x, y, 0)=T^{0}(x, y),
$$

Boundary conditions on $x$ axis are:

$$
T(0, y, t)=\mu_{0}(y, t), T\left(l_{x}, y, t\right)=\mu_{t}(y, t)
$$

and on $y$ axis are:

$$
\begin{aligned}
& \theta_{0} \frac{\partial T(x, 0, t)}{\partial y}+\eta_{0} T(x, 0, t)=\varphi(x, t) \\
& \theta_{1} \frac{\partial T\left(x, l_{y}, t\right)}{\partial y}+\eta_{1} T\left(x, l_{y}, t\right)=\psi(x, t)
\end{aligned}
$$

The task is to develop a numerical method that ensures the consistency of solutions in two coordinate directions.

\section{Methods}

2.1 Differential-difference method for solving the problem 
A uniform grid on $x$ and $y$ is introduced

$$
\begin{aligned}
& \omega_{x}=\left(x_{i}=i h_{x}, i=0, \ldots, N_{x}+1 ; \quad h_{x}=\frac{l_{x}}{N_{x}+1}\right) \\
& \omega_{y}=\left(y_{i}=j h_{y}, j=0, \ldots, N_{y}+1 ; h_{y}=\frac{l_{y}}{N_{y}+1}\right)
\end{aligned}
$$

and a uniform or non-uniform time step $\tau_{n}=t_{n}-t_{n-1}$.

The grid sought for function $u_{i, j}^{n}$ and functions $f_{i, j}^{n}, \mu_{0, j}^{n}, \mu_{l, j}^{n}, \ldots$ are introduced.

First, for a fixed value of $j\left(0<j<N_{y}+1\right)$, the equation is approximated for internal nodes on $x$ with the second order of accuracy:

$$
\frac{\partial u_{i, j}^{n}}{\partial t}=\frac{a^{2}}{h_{x}^{2}}\left(u_{i+1, j}^{n}-2 u_{i, j}^{n}+u_{i-1, j}^{n}\right)+a^{2} \frac{\partial^{2} u_{i, j}^{n}}{\partial y^{2}}+f_{i, j}^{n}
$$

In special cases, $i=1$ and $i=N_{x}$ the boundary conditions of the problem are realized:

$$
\begin{gathered}
\frac{\partial u_{1, j}^{n}}{\partial t}=\frac{a^{2}}{h_{x}^{2}}\left(u_{2, j}^{n}-2 u_{1, j}^{n}+\mu_{0, j}^{n}\right)+a^{2} \frac{\partial^{2} u_{1, j}^{n}}{\partial y^{2}}+f_{1, j}^{n}, \\
\frac{\partial u_{N_{x}, j}^{n}}{\partial t}=\frac{a^{2}}{h_{x}^{2}}\left(\mu_{l, j}^{n}-2 u_{N_{x}, j}^{n}+u_{N_{x}-1, j}^{n}\right)+a^{2} \frac{\partial^{2} u_{N_{x}, j}^{n}}{\partial y^{2}}+f_{N_{x}, j}^{n} .
\end{gathered}
$$

The last three notations are written in matrix form:

$$
\frac{\partial U_{j}^{n}}{\partial t}=\frac{a^{2}}{h_{x}^{2}} A U_{j}^{n}+a^{2} \frac{\partial^{2} U_{j}^{n}}{\partial y^{2}}+F_{j}^{n},
$$

where

$$
\begin{gathered}
U_{j}^{n}=\left(u_{1, j}^{n}, u_{2, j}^{n}, \ldots, u_{N_{x}-1, j}^{n}, u_{N_{x}, j}^{n}\right)^{T} \\
F_{j}^{n}=\left(f_{1, j}^{n}+\frac{a^{2}}{h_{x}^{2}} \mu_{0, j}^{n}, f_{2, j}^{n}, \ldots, f_{N_{x}, j}^{n}+\frac{a^{2}}{h_{x}^{2}} \mu_{i, j}^{n}\right)^{T} \\
A=\left\|\begin{array}{cccccccc}
-2 & 1 & 0 & 0 & \cdots & 0 & 0 & 0 \\
1 & -2 & 1 & 0 & \cdots & 0 & 0 & 0 \\
0 & 1 & -2 & 1 & \cdots & 0 & 0 & 0 \\
& \cdots & & & \cdots & & \cdots & \\
0 & 0 & 0 & 0 & \cdots & -2 & 1 & 0 \\
0 & 0 & 0 & 0 & \cdots & 1 & -2 & 1 \\
0 & 0 & 0 & 0 & \cdots & 0 & 1 & -2
\end{array}\right\|_{N_{x}}
\end{gathered}
$$

Hereinafter $(\ldots)^{T}$ means the transposition of the matrix. 
Matrix $A$ is represented in the form [13]

$$
A=V \Lambda V^{-1}
$$

where the elements of the diagonal matrix $\Lambda$ have the form

$$
\lambda_{s}=-2\left(1+\cos \frac{\pi s}{N_{x}+1}\right)
$$

and the elements of fundamental matrix $V_{-}$

$$
v_{s, p}=(-1)^{s+p} \sqrt{\frac{2}{N_{x}+1}} \sin \frac{\pi s p}{N_{x}+1}
$$

$V^{-1}(=V)$ is the inverse $V$ matrix.

Multiplying both sides of equation (5) by $V^{-1}$ and, we get

$$
\frac{\partial V^{-1} U_{j}^{n}}{\partial t}=\frac{a^{2}}{h_{x}^{2}} V^{-1} A U_{j}^{n}+a^{2} \frac{\partial^{2} V^{-1} U_{j}^{n}}{\partial y^{2}}+V^{-1} F_{j}^{n}
$$

where the commutative property of differentiation and matrix multiplication operations was taken into account.

Introduce the new sought for vector-function $\bar{U}_{j}^{n}=V^{-1} U_{j}^{n}$, vector function $\bar{F}_{j}^{n}=V^{-1} F_{j}^{n}$, and consider the identity $V^{-1} A U_{j}^{n}=\Lambda \bar{U}_{j}^{n}[16]$.

Then equation (6) has the form:

$$
\frac{\partial \bar{U}_{j}^{n}}{\partial t}=\frac{a^{2}}{h_{x}^{2}} \Lambda U_{j}^{n}+a^{2} \frac{\partial^{2} \bar{U}_{j}^{n}}{\partial y^{2}}+\bar{F}_{j}^{n}
$$

which represents autonomous equations

$$
\frac{\partial \bar{u}_{i, j}^{n}}{\partial t}=\frac{a^{2}}{h_{x}^{2}} \lambda_{i} \bar{u}_{i, j}^{n}+a^{2} \frac{\partial^{2} \bar{u}_{i, j}^{n}}{\partial y^{2}}+\bar{f}_{i, j}^{n} .
$$

According to the introduced substitutions and identity $V^{-1}=V$, we get

$$
\begin{gathered}
\bar{u}_{i, j}^{n}=\sum_{p=1}^{N_{x}} v_{i, p} u_{p, j}^{n}, \\
\bar{f}_{i, j}^{n}=v_{i, j} \frac{a^{2} \mu_{0, j}^{n}}{h_{x}^{2}}+v_{i, N_{x}} \frac{a^{2} \mu_{i, j}^{n}}{h_{x}^{2}}+\sum_{p=1}^{N_{x}} v_{i, p} u_{p, j}^{n},
\end{gathered}
$$

Due to this substitution, the initial conditions change 


$$
\bar{u}_{i, j}^{0}=\sum_{p=1}^{N_{x}} v_{i, p} T_{p, j}^{0}
$$

and the boundary conditions change

$$
\begin{gathered}
\theta_{0} \frac{\partial \bar{u}_{i, j}^{n}}{\partial y}+\eta_{0} \bar{u}_{i, 0}^{n}=\bar{\varphi}_{i}^{n}=\sum_{p=1}^{N_{x}} v_{i, p} \varphi_{p}^{n} \\
\theta_{1} \frac{\partial \bar{u}_{i, N_{y}+1}^{n}}{\partial y}+\eta_{l} \bar{u}_{i, N_{y}+1}^{n}=\bar{\psi}_{i}^{n}=\sum_{p=1}^{N_{x}} v_{i, p} \psi_{p}^{n}
\end{gathered}
$$

For internal nodes on $j$, equation (7) is approximated by an implicit scheme, where the second order of accuracy is provided on the coordinate $y$ :

$$
\frac{\bar{u}_{i, j}^{n}-\bar{u}_{i, j}^{n-1}}{\tau_{n}}=\frac{a^{2}}{h_{x}^{2}} \lambda_{i} \bar{u}_{i, j}^{n}+\frac{a^{2}}{h_{x}^{2}}\left(\bar{u}_{i, j+1}^{n}-2 \bar{u}_{i, j}^{n}+\bar{u}_{i, j-1}^{n}\right)+\bar{f}_{i, j}^{n}
$$

Hence we find the values of sweep coefficients for $1 \leq j \leq N_{y}$

$$
\alpha_{j}=\frac{a_{j}}{b_{j}-c_{j} \alpha_{j-1}}, \quad \beta_{j-1}=\frac{c_{j} \beta_{j-1}-d_{j}}{b_{j}-c_{j} \alpha_{j-1}}
$$

The task is to determine the values of sweep coefficients at $y=0$, with which the direct sweep begins.

For the third kind of boundary conditions[19-23] $\left(\theta_{0} \neq 0, \eta_{0} \neq 0\right)$, at $y=0$ we ensure the approximation of the second order of accuracy and we get

$$
\alpha_{0}=\frac{\left(4 a_{1}-b_{1}\right) \theta_{0}}{\left(3 a_{1}-c_{1}\right) \theta_{0}-2 h_{y} a_{1} \eta_{0}}, \beta_{0}=\frac{-\theta_{0} d_{1}-2 a_{1} h_{y} \bar{\varphi}_{i}^{n}}{\left(3 a_{1}-c_{1}\right) \theta_{0}-2 h_{y} a_{1} \eta_{0}}
$$

According to the obtained values of $\alpha_{j}, \beta_{j}$ and boundary conditions, the values of $\bar{u}_{i, N_{y}+1}^{n}$ are determined.

At $y=l_{y}$ the condition of the third kind, the second condition from (4) at $\theta_{1}=1, \eta_{l} \neq 0$ is approximated by the second order of accuracy.

As a result, we obtain 


$$
\bar{u}_{i, N_{y}+1}^{n}=\frac{2 h_{y} \bar{\psi}_{i}^{n}-\theta_{i}\left[\beta_{N_{y-1}}-\left(4-\alpha_{N_{y}-1}\right) \beta_{N_{y}}\right]}{2 h_{y} \eta_{l}-\theta_{l}\left[3-\left(4-\alpha_{N_{y}-1}\right) \alpha_{N_{y}}\right]}
$$

Direct and reverse sweeps are performed for sections $i=1 . . N_{x}$.

Reverse transition to $u_{i}^{n}$, taking into account the identity $V=V^{-1}$, at $i=1 . . N_{x}$ and $j=0 . . N_{y}+1$ is carried out according to the formula

$$
u_{i, j}^{n}=\sum_{p=1}^{N_{x}} v_{i, p} \bar{u}_{p, j}^{n}
$$

\section{Results and Discussion}

\subsection{An algorithm for solving an elliptic equation}

The above algorithm can be used to solve the elliptic equation with the introduction of fictitious time (or by the method of successive approximation) [13]. These complications, as noted above, are due to the inconsistency of the values sought from neighboring layers used in the finite difference equations.

When solving an elliptic equation

$$
a^{2}\left(\frac{\partial^{2} T}{\partial x^{2}}+\frac{\partial^{2} T}{\partial y^{2}}\right)+f(x, y, t)=0
$$

where the right-hand side is a function of time, the method proposed above can be applied.

After introducing $\bar{U}_{i, j}^{n}$ and approximating on y, the finite difference equations take the form

$$
\frac{\lambda_{i}}{h_{x}^{2}} \bar{u}_{i, j}^{n}+\frac{\bar{u}_{i, j+1}^{n}-2 \bar{u}_{i, j}^{n}+\bar{u}_{i, j-1}^{n}}{h_{y}^{2}}=-\frac{1}{a^{2}} \bar{f}_{i, j}^{n} .
$$

If we introduce the notation $\sigma=h_{y}^{2} / h_{x}^{2}$, then the finite difference equations take the form

$$
\bar{u}_{i, j+1}^{n}-\left(2-\sigma \lambda_{i}\right) \bar{u}_{i, j}^{n}+\bar{u}_{i, j-1}^{n}=-\frac{h_{y}^{2}}{a} \bar{f}_{i, j}^{n}
$$

According to this notation, the sweep coefficients are determined by formulas 


$$
\alpha_{j}=\frac{1}{2-\sigma \lambda_{i}-\alpha_{j-1}}, \beta_{j-1}=\frac{\beta_{j-1}+h_{y}^{2} \bar{f}_{i, j}^{n} / a^{2}}{2-\sigma \lambda_{i}-\alpha_{j-1}}
$$

for $1 \leq j \leq N_{y}$ at a given value of $i=\left[1 . . N_{x}\right]$.

The boundary conditions of the problem are realized in the order given above.

As seen, the second order of accuracy on $h_{x}$ and $h_{y}$ is ensured. But the main advantage is an elimination of discrepancies in approximation on two coordinates. Accordingly, for a fixed value of $t$, the algorithm is used only once. If the right side of the equation does not depend on time, then a one-time use of the algorithm leads to the goal.

\subsection{Computational experiment}

Based on the presented algorithms, the programs in the Pascal ABC environment were drawn up, and calculations were carried out for various heat transfer problems.

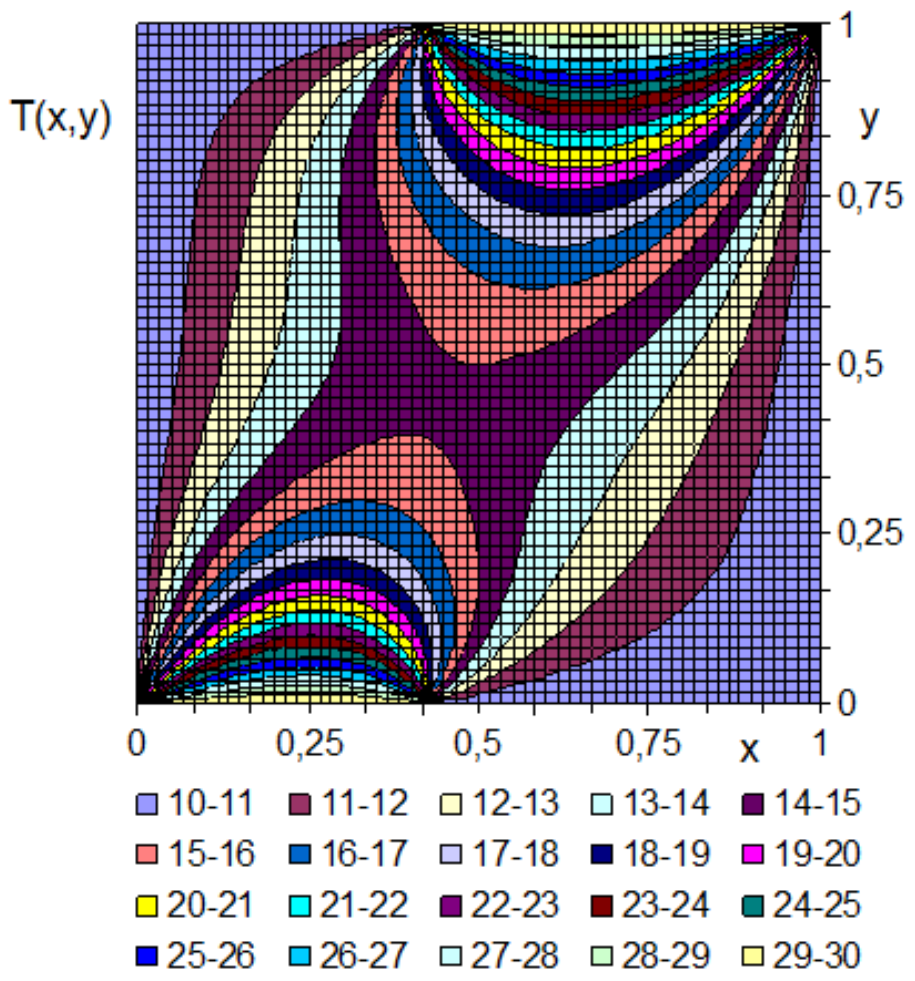

Fig. 1. The field of isotherms obtained using the differential-difference method for discontinuous boundary functions

$$
\mu_{0}(y)=\mu_{l}(y)=10 \text { at }\left((x=0) \cup\left(x=l_{x}=1\right)\right) \cap\left(0 \leq y \leq l_{y}=1\right),
$$




$$
\phi(x)=\left\{\begin{array}{lll}
30 & \text { at } & 0 \leq x \leq 5 / 12 \\
10 & \text { at } & 5 / 12 \leq x \leq 1 ;
\end{array} \quad \psi(x)=\left\{\begin{array}{lll}
10 & \text { at } & 0 \leq x \leq 5 / 12 \\
30 & \text { at } & 5 / 12 \leq x \leq 1
\end{array}\right.\right.
$$

Test problems for the calculation algorithm of the elliptic equation at the sought for functions $T(x, y)=x^{2}+y^{2}+2$ and $T(x, y)=\left(x^{2}-1\right) \sin y$ showed good agreement (with the accuracy of $10^{-5}$ ) at $h_{x}=h_{y}=1 / 60$.

Applying the algorithm and program for discontinuous boundary conditions and a point source and heat outflow are shown in Figures 1 and 2.

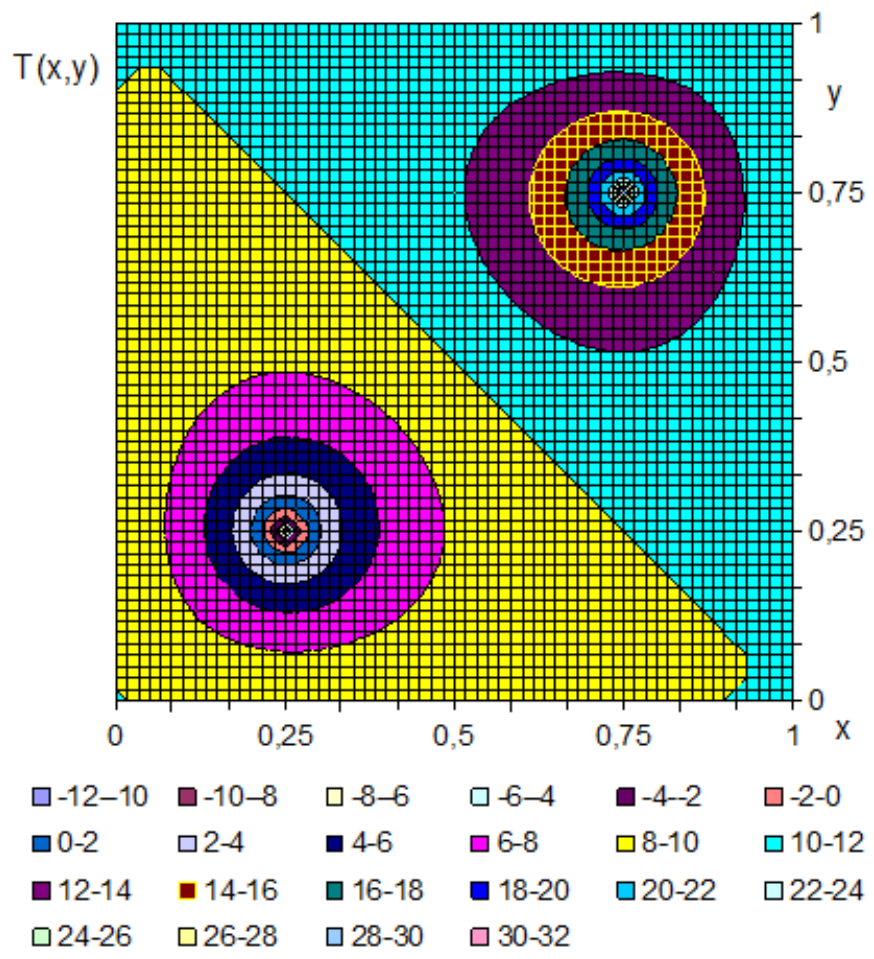

Fig. 2. Isotherms obtained when solving equation

$$
\frac{\partial^{2} T}{\partial x^{2}}+\frac{\partial^{2} T}{\partial y^{2}}=100000[\delta(0.25 ; 0.25)-\delta(0.75 ; 0.75)] \text { at } T_{G}=10
$$

A similar series of calculations were carried out using an algorithm and a program to solve a parabolic equation [20].

The first series of non-stationary heat transfer calculations refer to the method of successive approximations when the heat transfer equation has a homogeneous form

$$
\frac{\partial T}{\partial t}=a^{2}\left(\frac{\partial^{2} T}{\partial x^{2}}+\frac{\partial^{2} T}{\partial y^{2}}\right)
$$


At zero initial conditions:

$$
T(x, y, 0)=0
$$

At $x=0$ and $x=1$ boundary conditions do not depend on longitudinal coordinate:

$$
\begin{aligned}
& T(0, y, t)=\mu_{0}(y, t)=5 \sin \pi t, \\
& T(1, y, t)=\mu_{l}(y, t)=2 \sin 2 \pi t .
\end{aligned}
$$

At $y=0$ and $y=1$ the conditions of "no leakage" of heat are imposed:

$$
\frac{\partial T(x, 0, t)}{\partial y}=\varphi(x, t)=0, \quad \frac{\partial T(x, 1, t)}{\partial y}=\psi(x, t)=0
$$

Under given conditions, a solution is expected that does not depend on the longitudinal coordinate.

Calculations for this problem were carried out in a square area $1 \times 1$ at values of grid method indices of $\tau=0.02, h_{x}=h_{y}=0.02, N_{x}=N_{y}=49$. After the $5000^{\text {th }}$ time step, the difference between subsequent periods, and the analytical solution, did not exceed $10^{-5}$.

The same comparison results were obtained when solving the problem with discontinuous boundary conditions.

In the process of computational experiment on the last problem, the following feature was observed. 


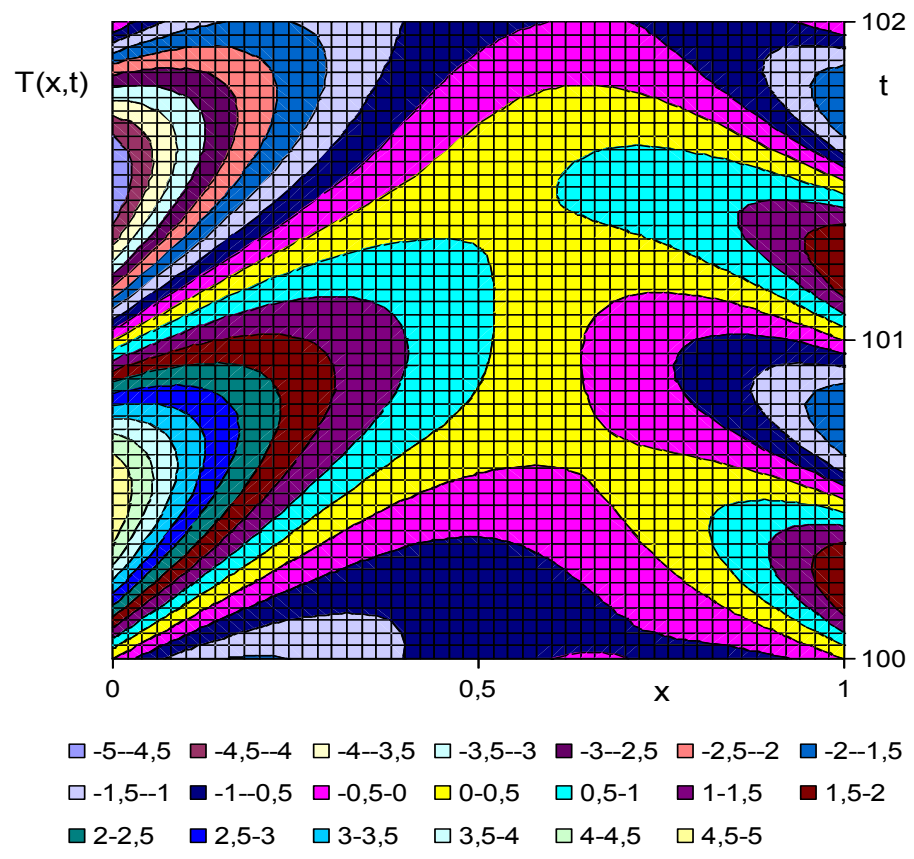

Fig. 3. Isotherms on coordinates $(x, t)$ obtained for $a^{2}=0.1 \mathrm{~m}^{2} /$ sek,

$$
T(0, y, t)=5 \sin \pi t, T(1, y, t)=2 \sin 2 \pi t, \frac{\partial T(x, 0, t)}{\partial y}=0, \frac{\partial T(x, 1, t)}{\partial y}=0
$$

The same comparison results were obtained when solving the problem with discontinuous boundary conditions.

In the process of computational experiment on the last problem, the following feature was observed.

To ensure approximation accuracy (maximum deviation of temperature values in subsequent approximations) of 0.001, 7065 approximations were required. At $\tau=0.2,1,5$, 25 , and 100 , when the approximation accuracy was 0.0001 , the approximations were 1877 , $461,113,31$ and 11 . Here, beginning from $\tau=5$, the maximum deviation of results from the numerical solution of the elliptic equation increased.

The second series of calculations refers to intensifying the process of heat exchange with the moving contact of a high-temperature body, which shows a more uniform temperature distribution. 


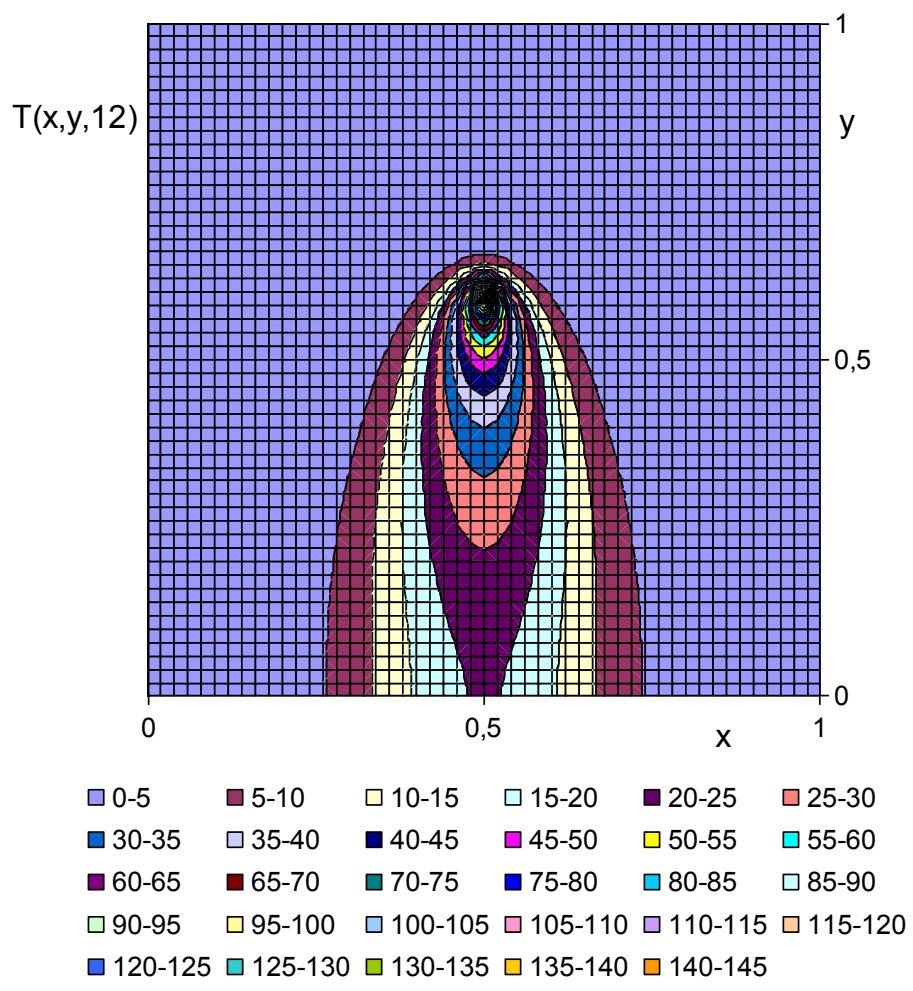

Fig. 4. Isotherms at a point of time $t=12 . l_{x}=1, l_{y}=1, N_{x}=N_{y}=49$, $\tau=0.02, a^{2}=0.001 \mathrm{~m}^{2} / \mathrm{sek}, U=0.05 \mathrm{~m} / \mathrm{sek}, \theta_{0}=\theta_{l}=1, \quad \xi_{0}=\xi_{l}=0, \quad q=1000.0$.

The last series of calculations refer to the problem with moving heat sources inside the heat transfer region, modeled by the equation

$$
\frac{\partial T}{\partial t}=a^{2}\left(\frac{\partial^{2} T}{\partial x^{2}}+\frac{\partial^{2} T}{\partial y^{2}}\right)+\delta\left[x_{0}(t), y_{0}(t)\right] q(t),
$$

where $\delta(\ldots)$ is the Dirac delta function, $q(t)$ is the heat release rate at a point $\left(x_{0}, y_{0}\right)$ , given by the density and heat capacity of the heat-conducting material.

The calculations were performed for the rectilinear (Fig. 4) and circular trajectories of point sources. In calculations, the source location $\left(x_{0}, y_{0}\right)$ was replaced by the closest grid node. 


\section{Conclusions}

Using straight lines and ordinary sweep methods, an exact analytical method has been developed to solve a system of finite difference equations followed from a certain class of two-dimensional problems based on elliptic and parabolic equations.

Applied approximation formulas ensure the second order of accuracy in spatial coordinates and the first order on time for a parabolic equation. The order of accuracy on time can be increased using the central scheme on time.

Some problems on the intensification of heat transfer with moving high-temperature elements and point heat sources have been solved; a method has been developed for the numerical implementation of a moving point source along an arbitrary path, the method has been checked for the rectilinear and circular motion of the source.

The developed method differs by the exact solution of finite difference equations and significantly reduces the calculation time. It can be used to solve a hyperbolic equation and three-dimensional linear partial differential equations.

The class of problems to be solved can be expanded using the results obtained in [1719], related to the mixed boundary conditions of the first and second kinds on the first coordinate.

\section{References}

1. Isaev S.I., Kozhinov I.A. et al. Theory of heat and mass exchange. Ed. A.I. Leontiev. M.: Higher School, 1979. - 495 p.

2. Lykov A.V., Mikhailov Yu.A. Theory of heat and mass transfer. - M.-L .: Gosenergoizdat, 1963.

3. Samarsky A.A., Vabishchevich P.N. Computational heat transfer. - M.: URSS, Editorial, 2009.- 784 p.

4. Ibragimov A.M. Unsteady heat transfer in building materials, products and structures under phase transformation of moisture. - Abstract. ... Doct.Tech. Sci. - Ivanovo, 2005 - $43 \mathrm{p}$.

5. Yang Chen, Qie Sun и Ronald Wennersten. Heat transfer characteristics of water during flow boiling in a vertical rectangular mini-channel / The $6^{\text {th }}$ International Conference on Applied Energy - ICAE2014, Energy procedia 61 (2014), pp. 109-112.

6. Wenke Zhang, Hongxing Yang, Lin Lu, Zhaohong Fang. The heat transfer analysis and optimal design on borehole ground heat exchangers / The 6th International Conference on Applied Energy - ICAE2014, Energy procedia 61 (2014), pp. 385-388.

7. Chungen Yin. Transient heating and evaporation of moving fuel droplets $/$ The $6^{\text {th }}$ International Conference on Applied Energy - ICAE2014, Energy procedia 61 (2014), pp. 37-40.

8. Tsoi P.V. Systemic methods for calculating boundary-value problems of heat and mass transfer: Direct and inverse problems of unsteady heat conduction and thermoelastic stresses. Hydrodynamics and heat transfer in the channels of a complex profile $/ 3^{\text {rd }}$ ed., revised and added. - M.: Publishing House MPEI, 2005 .- 568 p.

9. Tikhonov A.M., Samarsky A.A. Equations of mathematical physics. - M.: Nauka. 1977. - $736 \mathrm{p}$.

10. Pavlov A.R., Matveeva M.V. An iterative difference scheme for the problem of heat and mass transfer during freezing of soils. - Samara: Bulletin of SamSU, Natural Sciences, 2007, No. 6 (56). - P. 242-253.

11. Prikhodko A.A., Alekseenko S.V. Mathematical modeling of unsteady processes during freezing and thawing of porous media // Collection of scientific articles: Modern Science, 2012, No. 2 (10). - P. 57-63. 
12. Mazhukin V.I. Mathematical modeling of the Stefan problem on an adaptive grid. Problem reports of the Minsk International Forum "Heat and Mass Transfer", May 2427, 1988, Minsk, Institute of Heat and Mass Transfer named after A.V. Lykov. - P. 125-139.

13. Paskonov V.M., Polezhaev V.I., Chudov L.A. Numerical modeling of heat and mass transfer processes. - M.: Nauka, 1984. - 288 p.

14. Anderson D., Tannehill J., Pletcher R. Computational hydromechanics and heat transfer: In 2 vols. Translated from English. - M.: Mir, 1990 .- P. 728 (1st volume, 392 p.).

15. Faddeeva V.N. The straight lines method as applied to some boundary value problems. - Reports of MI of the Academy of Sciences of the USSR, 1949, Volume 28 .- P. 73-103. (From the All-Russian Mathematical Portal Math-Net).

16. Karimberdieva S. Numerical methods for solving differential-difference equations in a parallelepiped, a sphere and a cylinder. - Tashkent: Fan, 1983.- 112 p.

17. Karimov I.K., Khuzhayev I.K., Khuzhayev Zh.I. The use of the straight line method for solving a one-dimensional equation of parabolic type under boundary conditions of the second and first kinds // News of KRAUNTS, 2018, 1 (21). - P. 78-93.

18. Aliev F.A., Khuzhayev Zh.I., Ravshanov Z.N. The differential-difference method for solving one-dimensional equations of parabolic type under boundary conditions of the first and second kinds // Scientific News of Andijan State University. - Andijan, 2017.- No.4.- P.5-10.

19. Abdullayev, A.A., Ergashev, T.G. Poincare-tricomi problem for the equation of a mixed elliptico-hyperbolic type of second kind. Vestnik Tomskogo Gosudarstvennogo Universiteta, Matematika i Mekhanika, 2020, (65), P. 5-21, DOI 10.17223/19988621/65/1

20. Islomov B. I. Abdullayev A.A. On a problem for an elliptic type equation of the second kind with a conormal and integral condition. Nanosystems: Physics, Chemistry, Mathematics, 2018, 9 (3), P. 307-318 DOI 10.17586/22208054201893307318

21. Abdullaev A.A. M Hidoyatova Innovative distance learning technologies. Journal of Critical Reviews 2020; 7(11): 337-339 http://www.jcreview.com/?mno=117623 DOI: $10.31838 /$ jcr.07.11.57

22. V. Vahobov, A.A.Abdullayev, Kh. Kholturayev, M. Hidoyatova, A. Raxmatullayev On asymptotics of optimal parameters of statistical acceptance control. \ Journal of Critical Reviews 2020, 7 (11), 330-332 DOI: http://dx.doi.org/10.31838/jcr.07.11.55

23. T. K. Yuldashev, B. I. Islomov, A. A. Abdullaev. On solvability of a PoincareTricomi Type Problem for an Elliptic-hyperbolic Equation of the Second Kind. Lobachevskii Journal of Mathematics, 2021, Vol. 42, No. 3, pp. 662-674. DOI: $10.1134 /$ S1995080221030239 